\title{
Research of Wireless Sensor Network Nodes based on ZigBee
}

\author{
Liu Chunguang \\ Editorial Department of Journal \\ Shenyang Jianzhu University \\ Shenyang, China
}

\author{
Zhu Huijie \\ Finance Department \\ Shenyang Jianzhu University \\ Shenyang, China
}

Wang Xin

School of Information \& Control Engineering

Shenyang Jianzhu University

Shenyang, China

Liu Xiyang

School of Information \& Control Engineering Shenyang Jianzhu University

Shenyang, China

\author{
Yao Jinmei* \\ Postgraduate School \\ Shenyang Jianzhu University \\ Shenyang, China \\ e-mail:arnold0110@sina.com \\ * Corresponding Author \\ Xu Yumei \\ Editorial Department of Journal \\ Shenyang Jianzhu University \\ Shenyang, China
}

\author{
Mao Yongming \\ School of Information \& Control Engineering \\ Shenyang Jianzhu University \\ Shenyang, China
}

\author{
Wang Bin \\ Northeastern University at Qinhuangdao \\ Qinhuangdao, China
}

\begin{abstract}
There is a large prospect of application for wireless sensor network based on ZigBee. Design a lowpower network node, reducing the losing of the datagram and avoiding the clash between the data is the key for improving the lifecycle of the network node. For resolving the above problem effectively, the relation between power consumption and data traffic of physical layer, the relation between the power consumption and system throughput, the relation between distance of communications and Packet Loss Rate of MAC and the relation between distance of communications and sensitivity are simulated from physical layer and MAC of the communication layer of IEEE802.15.4. Reducing the data traffic of nodes is the most effective plan on resolving energy efficient; the losing of the datagram could be reduced by short-distance transmission at the time of the data of nodes transmission; increasing the interval of sending packets and transmission delay of data transmission of nodes could avoid the clash between large amounts of data. The results show that short distance, large interval of sending packets and long transmission delay are appropriate for the transmission of large amounts of data of nodes. The results are very helpful for resolving the energy conservation of wireless sensor network node, the losing of the datagram and the clash between large amounts of data.
\end{abstract}

Keywords- ZigBee; MAC; Wireless sensor network; Physical layer; IEEE802.15.4

\section{INTRODUCTION}

$\mathrm{ZigBee}^{[1,2]}$ is a new wireless network technology for its short range, low speed, low power consumption and low cost. It is a technology solution between the wireless technology and Bluetooth. It uses a direct sequence spread spectrum (DSSS) technology of working frequency with $868 \mathrm{MHz}, 915 \mathrm{MHz}$ and $2.4 \mathrm{GHz}$. The frequency of DSSS doesn't need to apply the licences. It is adapted to the communications data of small volume and the occasion of lower data transfer efficiency. The security and reliability of data have a set of requirements, and its cost and power consumption are very low. Therefore, ZigBee has broad application prospects in industrial control, automation of industrial wireless positioning, home networking, automotive, medical equipment control and other fields in the next few years ${ }^{[3-9]}$. ZigBee chips will be the main field of application for home automation and industrial control in the future. Because of the small of technical research and the insufficiency of related data, the ZigBee technology has not been universal in our country. This paper simulate the relation between the power consumption and data traffic and the relation between the power consumption and system throughput for physical layer for the sensor network node and the further research has been done about the losing of datagram, the clash between the data and the power consumption in the process of node data transmission of MAC, at the same time extending the life cycle of the network. The results will be great reference value for understanding research of wireless sensor network nodes based on ZigBee. 


\section{ZIGBEE TECHNOLOGY}

\section{A. ZigBee overview}

Maintaini ZigBee originated from the dance of the ZigZag shape for colonies to jump and notify information about the other pollen of bees on the location to achieve the purpose of communication with each other, as a name of the new generation of wireless communication technology. ZigBee is a new wireless network technology for its short range, low speed, low power consumption and low cost. It is a technology solution between the wireless technology and Bluetooth. It is adapted to the communications data of small volume and the occasion of lower data transfer efficiency. The security and reliability of data have a set of requirements, and its cost and power consumption are very low. IEEE handle low-level MAC layer and physical layer protocol, ZigBee Alliance standardized network layer protocol and APL and develop a security layer to guarantee that this portable device is not accidentally disclosed and the data of relying on network long distance transmission will not achieved other node.

\section{B. ZigBee network architecture}

Wherever Times is specified, Times Roman or Times New Roman may be used. If neither is available on your word processor, please use the font closest in appearance to Times. Avoid using bit-mapped fonts if possible. TrueType 1 or Open Type fonts are preferred. Please embed symbol fonts, as well, for math, etc.

a. ZigBee network devices

The device mainly consists of network coordinator, full-function device (FFD) and reduced-function device (RFD). Network Coordinator includes the messages from all networks, the largest of storage capacity and the strongest of calculation capability. The function of network coordinator is to send a beacon, establish a network, manage the network nodes, store the information for network node, search for routing messages of a pair of nodes, and receive the information continuously. FFD can act as network coordinator, forming the networks of connecting other FFD and RFD. The function of controller can provide with bi - directional transmission of the information. RFD can only transmit information to the FFD and receive information from FFD.

b. ZigBee networks topology and the node type

There are 3 types of structures for the networks topology in ZigBee wireless networks: the star network topology, the tree network topology and the mesh network topology. The structure of the topology of wireless sensor networks is shown as Fig.1.
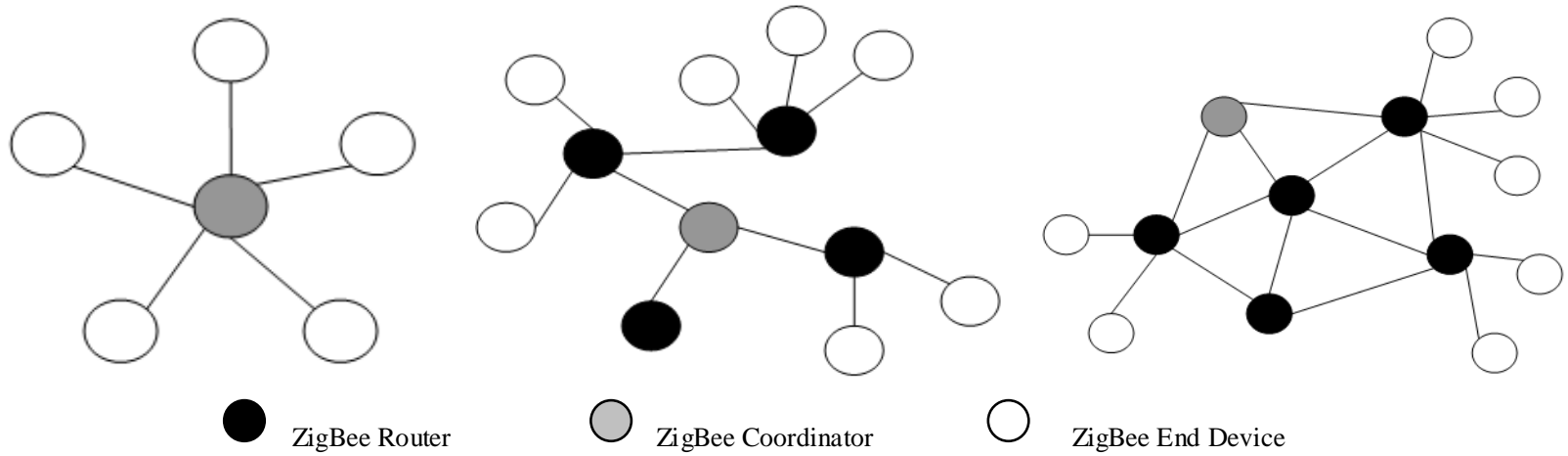

Figure 1. Structure of the topology of wireless sensor networks:(a) Star network topology;(b) Tree network topology;(c) Mesh network topology

From the network configuration, there are 3 types of ZigBee network nodes: ZigBee Coordinator, ZigBee Router and ZigBee End Devices.

ZigBee coordinator can be brought together in the wireless sensor network node. ZigBee coordinator must be the type of FFD. ZigBee network only have a ZigBee coordinator. Collector point can maintain the indirect addressing used bound forms, and it also can perform and relate to other tasks. ZigBee Router must also be the FFD. The data forwarding node of ZigBee router can forward the received data to the other node. ZigBee End Devices can be the FFD or the RFD, it connects to the network through ZigBee Coordinator and ZigBee Router, but it doesn't joined the network through any other nodes, ZigBee End Devices can run at a very low power. c.Characteristic

With the deepening of ZigBee technology, its advantages stand out continuously, mainly in the follows:

(1) Power saving. The two sections of AA battery support usage time of six months to two years time. (2) Reliable. Use collision avoidance mechanisms while reserving a dedicated time slot for requiring a fixed bandwidth of the communication service to avoid competing and conflicting of the sending data. The nodes module have the function of automatic dynamic networks between them, information is transferred by means of automatic routing in ZigBee throughout network to ensure the reliability of the information transmission. (3) Short delay. Optimized for the applications of delay - sensitive, communication delays and activate from sleep the delay are very short. (4) Network capacity. It supports 65,000 nodes. (5) Security. ZigBee provides data confidentiality and security keys, the algorithms of encryption use the general Advanced Encryption Standard (AES).

\section{ZIGBEE PROTOCOL STACK}

ZigBee protocol stack consists of a set of sublayers, each layer provides a specific set of services for its upper class; A data entity provides the services of data transmission, and a management entity provides the other services. Each service entity provides service interfaces for upper by SAP, and each SAP provides a set of basic 
services directive to implement the commensurable

The standard of IEEE802.15.4-2003 defines two layers at the bottom: physical layer (PHY) and medium access control (MAC) sublayers. ZigBee provides the design of the framework of network layer and application layer (APL), its APL framework includes application support sublayer (APS ), ZigBee device objects (ZDO) and the objects of the application of the manufacturer.

\section{A. Communication layer of IEEE802.15.4}

The architecture of ZigBee protocol stack is shown in Fig.2. The standard of IEEE802.15.4 defines two layers at the bottom: Physical layer (PHY) and Media Access Control (MAC) Sublayers. ZigBee use directly physical layer and media access control sublayers, which are defined by IEEE802.15.4.

a.Physical layer (PHY)

According to different countries and regions, communication frequency of ZigBee provides different operating frequency range in the specification of physical layer, the frequency range of Zigbee are respectively $2.4 \mathrm{GHz}$ and $868 / 915 \mathrm{MHz}$. Therefore, IEEE802.15.4 defined two standards of physical layer, which are respectively ( namely) $2.4 \mathrm{GHz}$ physical layer and $868 / 915 \mathrm{MHz}$ physical layer. Two physical layers are based on direct sequence spread spectrum (DSSS) technology. The band defined 27 channels of physical

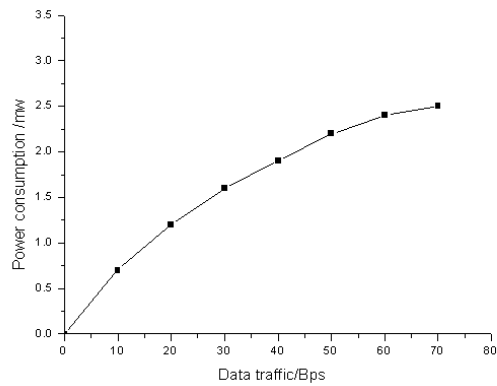

function.

band in the IEEE802.15.4. The band of $868 \mathrm{MHz}$ defines a channel; The band of $915 \mathrm{MHz}$ defines 10 channels, channel spacing is $2 \mathrm{MHz}$; The band of $2.4 \mathrm{GHz}$ defines 16 channel, channel spacing is $5 \mathrm{MHz}$. The center frequency of channel is defined as follow ( $\mathrm{k}$ is the number of channels ):

$$
f_{c}=\left\{\begin{array}{l}
868.3 \mathrm{MHz}, \quad k=0 \\
906 \mathrm{MHz}+2(k-1) \mathrm{MHz}, k=1,2, \cdots, 10 \\
2401 \mathrm{MHz}+5\left(k_{\text {the }}-11\right) \mathrm{MHz} \text { bands } 1,12, \ldots \text { avairability }
\end{array}\right.
$$
overcrowding and the data rate, the IEEE802.15.4 selects a channel in the 27 channels. Judging from the energy, cost and efficiency, different data rates can provide a better choice for different applications.

In order to reduce the power consumption of physical layer protocol, this paper simulates the relation between data traffic and the power consumption for the physical layer protocol and the relation between system throughput and the power consumption for the physical layer protocol with Matlab7.1. The further research has been done about the relation between data traffic and the power consumption and the relation between system throughput and the power consumption. The result is shown as Fig.2.

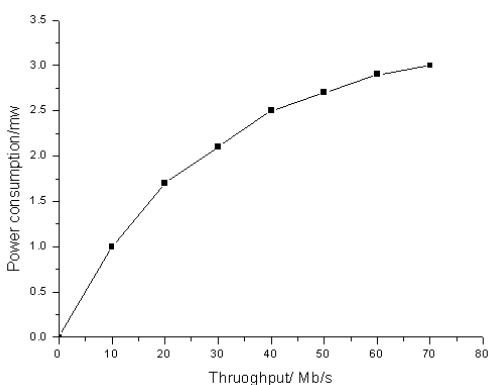

Figure 2. (a) The relation between the data flows and the power consumption for the physical layer protocol; (b) The relation between the system throughput and the power for the physical layer protocol

The result shows that the power consumption is low at the time of reducing the data traffic by comparing the relation between data traffic and power consumption with the relation between throughput and power consumption. So the most fundamental solutions of energy saver of physical layer are reducing the data traffic. Reducing the data traffic is lower than the reducing throughput, and the reason is that the nodes spend too much time with waiting for packet arrival. Power consumption of during to idle listening can be saved by maintaining while transceivers are closed. In addition, when the node has no data of receiving and sending, the method of going into hibernation and turning off RF module can reduce energy waste.

b.Media Access Control Sublayers, MAC

MAC Protocol for wireless sensor networks is responsible for the establishment, the maintenance and the control to reducing the clash of neighboring node broadcasts. The focus of the study for WSN is about the performance of network has a greater impact for concerning about the quality of service and resolving the problem effectively. MAC Protocol for wireless sensor network is not only maximizing to throughput and minimizing to delay, it is the most important that the further research has been done about the losing of datagram, the clash between the data and the power consumption in the process of node data transmission of MAC, at the same time extending the life cycle of the network. Therefore, this paper researches the above problem in-depth study.

(1)Cause of losing of data traffic

For example, when the node has 1000 packets of a sending looping, the relation between sending and receiving packets rate and communication distance as shown in table 1.

TABLE I THE RELATION BETWEEN SENDING AND RECEIVING PACKETS RATE AND COMMUNICATION DISTANCE

\begin{tabular}{|c|c|c|c|}
\hline distance (m) & Sent packets & $\begin{array}{c}\text { Received } \\
\text { packets }\end{array}$ & $\begin{array}{c}\text { Packet Loss } \\
\text { Rate (\%) }\end{array}$ \\
\hline 5 & 1000 & 997 & 0.3 \\
\hline 10 & 1000 & 995 & 0.5 \\
\hline 15 & 1000 & 904 & 0.96 \\
\hline 20 & 1000 & 782 & 21.8 \\
\hline 25 & 1000 & 215 & 78.5 \\
\hline
\end{tabular}


From the above figure, the figure shows that the Packet Loss Rate will increase with the distance of communications, but with the distance of communications increasing the sensitivity will be reduced. Within the communication distance is less than 15 meter, packet loss rate is very small and communications are relatively reliable the relationship between the nodes. The packet loss increases obviously at around 20 meters. When the

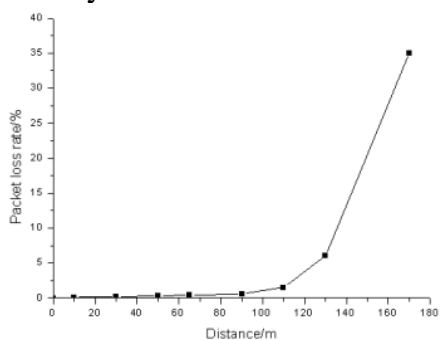

distance is increasing to 25 meters, communication has become notoriously unreliable the relationship between the nodes. The reason is that the communication distance is too far. In order to further verify the impact of the communication distance, this paper simulates the relation between distance of communications and Packet Loss Rate and the relation between distance of communications

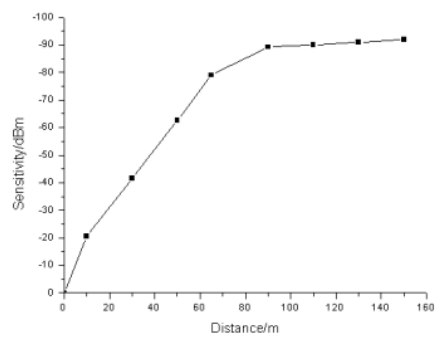

Figure 3. (a) The relation between distance of communications and Packet Loss Rate; (b)The relation between distance of communications and sensitivity

and sensitivity with Matlab7.1. The result is shown as Fig.3.

The result shows that the relation between distance of communications and Packet Loss Rate and the relation between distance of communications and sensitivity have been simulated, and the results show that the Packet Loss Rate will increase with the distance of communications, and the reason is that the original topology changes because of the communication distance is too long, and leading to lose data packet. With the distance of communications increasing the sensitivity will be reduced, and the reason is that it uses the method of two nodes sending the data each other when the communication distance is relatively far, and without any obstacle between the two nodes. Therefore, the sensitivity reduces gradually.

\section{CONCLUSIONS}

(a) The power consumption is low at the time of reducing the data traffic by comparing the relation between data traffic and power consumption with the relation between throughput and power consumption. So the most fundamental solutions of energy saver of physical layer is reducing the data traffic.

(b) The relation between distance of communications and Packet Loss Rate and the relation between distance of communications and sensitivity have been simulated, and the results show that the Packet Loss Rate will increase with the distance of communications, but with the distance of communications increasing the sensitivity will be reduced. So short-distance transmission could reduce the losing of data packet when the data of nodes starts to transfer.

(c) The relation between the interval of sending packets and the power consumption and the relation between transmission delay and the power consumption have been simulated, and the results show that the power consumption will get lower with the increasing of interval of sending packets and transmission delay. So the clash between the large amounts of data of nodes would be avoided by increasing the interval of sending packets and transmission delay.

So the results are very helpful for resolving the energy conservation of wireless sensor network node, the losing of the datagram and the clash between large amounts of data.

\section{REFERENCES}

[1] WH Li, CY Duan. The entrance and practice for ZigBee wireless network technology, Beijing : Press of Beijing University of Aeronautics and Astronautics,2007.

[2] Wang Xin, Huang Kuan, Gao Zhijun. The Primary Users' Signals Recognition Algorithm in Cognitive Radio Networks via KPCA and Random Forest[J].ICIC Express Letters,2015, 9(4):1083-1088.

[3] GB Wu, CY Liang. Research of energy-efficiency for wireless sensor networks. Journal of Transducer Technology, 2004, 23(7):74-76.

[4] F Bai, XF Yu, YL Yang. Research on energy-saving technology of ZigBee networks. Ordnance Industry Automation, 2008, 27(10): 7880.

[5] LM Sun, JH Li. Wireless sensor networks. Beijing: Tsinghua University Press, 2005,5: 7-8.

[6] Wang Xin,Guo Lili,Ma Lina. Cooperative Spectrum Sensing Algorithm Based on Second User Selection and Random Forest Classification[J].ICIC Express Letters, Part B: Applications,2015, 6(3): 845-850.

[7] J Zhao. Design and implementation of wireless sensor network node: Shandong University, 2006.

[8] DZ Zhang. Study on low power design technology for wireless sensor networks: Southwest University of Science and Technology, 2007.

[9] Wei Ye, J.Heidemann, D.Estrin. An Energy-Efficient MAC Protocol for Wireless Sensor Networks.In Proceeding of IEEE INFCOM,2002:1567-1576.

[10] JG Zhang, Wenbin LI, N Han,"Forest Fire Detection System Based on ZigBee Wireless Sensor Network," Journal of Beijing Forestry University,vol.694-697,2013,pp.961-965. 